\title{
Guidance Method for Mobile Manipulators Preventing Carrying Objects from Slipping
}

\author{
Masatoshi Hatano*, Mamoru Minami**, Toshiyuki Asakura** \\ and Tsuyoshi Ohsumi*
}

\author{
* Department of Mechanical and Inlellectual Sysiems Engineering, \\ Faculty of Engincering, Toyama University \\ 3190 Gofuku, Toyama, 930-8555, Japan \\ E-mail : masatosi@eng.toyama-u.ac.jp \\ - Department of Mechanical Engineering, Faculty of Engineering, Fukui University \\ 3-9-1 Bunkyo, Fukui, 910-8507, Japan \\ E-mail : minami@scmc.mech.fukui-u.ac.jp
}

\begin{abstract}
This research is concerned with a guidance method for mobile manipulators preventing carrying objects from slipping on a mobile manipulator while traveling. In this report, a new guidance method, in which translational and rotational accelerations for preventing slip are determined by considering analytically a mass and a position of a carrying object and friction forces, is proposed. In addition, it is shown that the our proposed method can navigate a mobile manipulator without slipping of a carrying object through real experiments.

Keywords : Mobile Manipulator, Construction Robot System, Nonholonomic, Guidance Control, Slip
\end{abstract}

\section{Introduction}

Mobile manipulators [1]-[5] are able to propel themselves around a working area and, unlike floor-fixed manipulators, are able to operate over a wide area. Basic functions of a mobile manipulator are classified roughly as follows; (1)a transporting function for a conveyance of objects, (2)a detecting function of a self position/orientation, (3) an operating function by a manipulator's hand. Focussing on the transporting function (1), it is generally known that navigating a mobile manipulator to a destination accurately is difficult [6][11], because a mobile manipulator travels under nonholonomic constraints [12]. Therefore, it is required to correct a position/orientation of the mobile manipulator while traveling in order to navigate it along a desired trajectory.

On the other hand, the surface of a table attached on a mobile manipulator for carrying objects should be flat, because an adjusted mechanism, which is for each shape of carrying objects for preventing them from slipping, restricts the shapes of the objects. Then, motions of the mobile manipulator's body while navigating cause slipping motions of carrying objects, when forces of inertia exerting the objects exceed static friction forces exerting them. Such slipping motions produce a load shifting while traveling and a positioning error of the objects by a mounted manipulator's hand at a operating point. In the present, a guidance method preventing carrying objects from slipping has not been proposed. The purpose of this research is to construct a guidance method preventing carrying objects from slipping for a mobile manipulator. Even when gains in a guidance controller which do not generate slipping motions are obtained, the controller produces slipping motions when more traveling errors occur than pre- liminary estimated errors caused by disturbances due to unknown shape of traveling terrain. In addition, when gains are set as small for occurrences of large unexpected traveling errors, the gains decrease performances of a guidance controller for small traveling errors.

In the present paper, a guidance method which restricts a present navigating quantity only when the force/torque exerting objects at one past control period exceeds a static friction force is proposed. In the method, navigating quantity not generating a slipping motion is determined analytically by considering a mass of the carrying object, a friction force between the object and a surface of a table for transporting and a position of the object on the table. The proposed method performs a guidance control in a range of limited translational and rotational accelerations which do not produce slipping motions. It is shown through real experiments that the proposed method can navigate a mobile manipulator with no slip of a carrying object.

\section{Guidance Method}

\subsection{Estimation of Position/Orientation}

In the present paper, it is assumed that a traveling plane is flat. $\Lambda$ traveling motion of a mobile robot, which is traveled and steered by two different driving wheels, in a two dimensional plane shown in Fig. 1(a) is considered. In the figure, $\boldsymbol{\Sigma}_{W}$ is a world frame, $\boldsymbol{\Sigma}_{M}$ is a frame of a mobile robot, $x$ and $y$ are positions of the origin of $\boldsymbol{\Sigma}_{M}$ referring to the origin of $\Sigma_{W}, V, \theta$ and $\omega$ are velocity, rotational angle and angular velocity of the center point between the two different wheels, respectively. Let velocities of $x$ and $y$ be $\dot{x}$ and $\dot{y}$, equations of traveling of the mobile robot shown in Fig. 1(a) are expressed by Eq.(1), respectively, on 


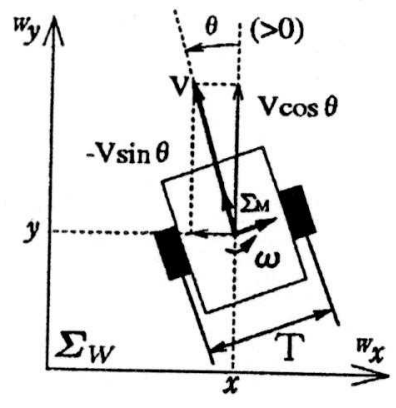

(a)Mobile robot

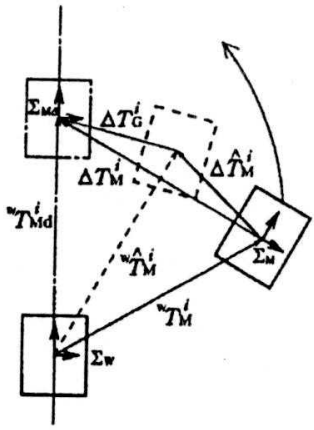

(b)Kinematics
Fig. 1 Image of guidance control

the assumption of no slip between driving wheels and a traveling plane.

$$
\dot{x}=-V \sin \theta, \quad \dot{y}=V \cos \theta, \dot{\theta}=\omega
$$

Then, a dead reckoning method is considered by using $x, y$ and $\theta$ which are estimated in real time. This method navigates the mobile robot by treating $\hat{x}, \hat{y}$ and $\hat{\theta}$ as feedback information. The $\hat{x}, \hat{y}$ and $\hat{\theta}$ are estimated values of $x, y$ and $\theta$ calculated by $\hat{V}$ and $\hat{\omega}$ which are estimated values of $V$ and $\omega$. Here, ${ }^{w} \boldsymbol{T}_{M}^{i}$ is a homogeneous matrix, which expresses the position/orientation of $\boldsymbol{\Sigma}_{W}$ referring to $\boldsymbol{\Sigma}_{W}$, and the estimated value ${ }^{W} \hat{\boldsymbol{T}}_{M}^{i}$ at time $i \Delta \tau$ is determined by:

$$
{ }^{W} \hat{\boldsymbol{T}}_{M}^{i}=\left[\begin{array}{cccc}
\cos \hat{\theta}^{i} & -\sin \hat{\theta}^{i} & 0 & \hat{x}^{i} \\
\sin \hat{\theta}^{i} & \cos \hat{\theta}^{i} & 0 & \hat{y}^{i} \\
0 & 0 & 1 & 0 \\
0 & 0 & 0 & 1
\end{array}\right]
$$

When Eq.(1) is integrated respect to time $t$ correctly, accurate $x, y$ and $\theta$ are estimated. Thus, Eq.(1) must be obtained within a control period $\Delta \tau$ for real time control. Therefore, integrated equations of Eq.(1) respect to time $t$ are approximated by [11]:

$$
\begin{aligned}
& \hat{x}^{i}=\hat{x}^{i-1}-\hat{V}^{i} \Delta \tau \sin \left\{\hat{\theta}^{i-1}+\left(\Delta \hat{\theta}^{i} / 2\right)\right\} \\
& \hat{y}^{i}=\hat{y}^{i-1}+\hat{V}^{i} \Delta \tau \cos \left\{\hat{\theta}^{i-1}+\left(\Delta \hat{\theta}^{i} / 2\right)\right\} \\
& \hat{\theta}^{i}=\hat{\theta}^{i-1}+\Delta \hat{\theta}^{i} \\
& \Delta \hat{\theta}^{i}=\hat{\omega}^{i} \Delta \tau
\end{aligned}
$$

Here, $\Delta \hat{\theta}^{i}$ and $\hat{V}^{i}$ are calculated actually by:

$$
\Delta \hat{\theta}^{i}=\left(L_{R}^{i}-L_{L}^{i}\right) / T, \hat{V}^{i}=\left(L_{R}^{i}+L_{L}^{i}\right) / 2 \Delta \tau(7)
$$

where $L_{j}^{i}=K_{j} P_{j}^{i}\left(j=L, R, K_{j}\right.$ is a coefficient and $P_{j}^{i}$ is a pulse count) is a traveled distance of the two driving wheels within $\Delta \tau$ and $T$ is tread. ${ }^{W} \hat{T}_{M}^{i}$ at time $i \Delta \tau$ is estimated by Eqs.(3) through

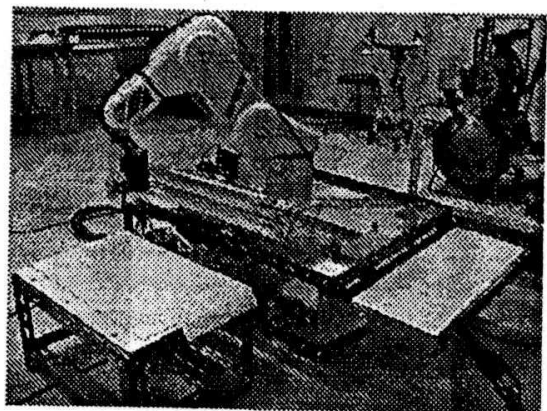

Fig. 2 Photo of a mobile manipulator

\subsection{Guidance Method}

A guidance method using Eqs.(3) through (6) is considered. A desired trajectory on a $x-y$ plane of $\boldsymbol{\Sigma}_{W}$ is given by $y(t)=f(x(t))$. Desired values of $x, y$ and $\theta$ at time $i \Delta \tau$ on a desired trajectory are expressed by $x_{d}^{i}, y_{d}^{i}$ and $\theta_{d}^{i}$ with the symbol " $i$ $"$. In addition, errors of the position/orientation of the mobile robot while navigating are denoted by $X_{e}^{i}, Y_{e}^{i}$ and $\theta_{e}^{i}$ which arc expressed with a frame on the desired trajectory and are obtained by:

$$
\left[\begin{array}{l}
X_{e}^{i} \\
Y_{e}^{i} \\
\theta_{e}^{i}
\end{array}\right]=\left[\begin{array}{ccc}
\cos \theta_{d}^{i} & \sin \theta_{d}^{i} & 0 \\
-\sin \theta_{d}^{i} & \cos \theta_{d}^{i} & 0 \\
0 & 0 & 1
\end{array}\right]\left[\begin{array}{c}
x_{d}^{i}-\hat{x}^{i} \\
y_{d}^{i}-\hat{y}^{i} \\
\theta_{d}^{i}-\hat{\theta}^{i}
\end{array}\right]
$$

The equations of guidance [6] for the mobile robot using $X_{e}^{i}, Y_{e}^{i}$ and $\theta_{e}^{i}$ are:

$$
\begin{aligned}
& V_{c}^{i+1}=V_{d}^{i} \cos \theta_{e}^{i}+k_{y} Y_{e}^{i} \\
& \omega_{c}^{i+1}=\omega_{d}^{i}+V_{d}^{i}\left(-k_{x} X_{e}^{i}+k_{\theta} \sin \theta_{e}^{i}\right)
\end{aligned}
$$

where $V_{d}^{i}$ and $\omega_{d}^{i}$ are a desired traveling speed and an angular velocity at time $i \Delta \tau, V_{c}^{i+1}$ and $\omega_{c}^{i+1}$ are a navigating traveling speed and an angular velocity between time $i \Delta \tau$ and $(i+1) \Delta \tau$, $k_{x}, k_{y}$ and $k_{\theta}$ are the gains of the guidance controller. Using this guidance control method given by Eq.(9) and Eq.(10), $X_{e}^{i}, Y_{e}^{i}$ and $\theta_{e}^{i}$ converge to zero when dynamics of the mobile robot can be ignored and the length of a traveling course is infinite.

\subsection{Kinematic Relationships of Guidance}

Fig. 1(b) shows kinematic relationships between a desired value ${ }^{W} \boldsymbol{T}_{M d}^{i}$, an actual value ${ }^{W} T_{M}^{i}$ and an estimated value ${ }^{W} \hat{\boldsymbol{T}}_{M}^{i}$ of the position/orientation of the mobile manipulator at time $i \Delta \tau .{ }^{i} T_{j}$ is a homogeneous matrix which expresses the position/orientation of $\boldsymbol{\Sigma}_{j}$ referring to $\boldsymbol{\Sigma}_{i}$. Using those values, an error $\Delta \hat{\boldsymbol{T}}_{M}^{i}$ of an estimation, an inner error $\Delta T_{G}^{i}$ and an external error $\Delta T_{M}^{i}$ of the guidance are defined by:

$$
\begin{gathered}
{ }^{W} T_{M d}^{i}={ }^{W} \hat{T}_{M}^{i} \Delta T_{G}^{i} \\
{ }^{W} T_{M d}^{i}={ }^{W} T_{M}^{i} \Delta T_{M}^{i} \\
{ }^{W} \hat{T}_{M}^{i}={ }^{W} T_{M}^{i} \Delta \hat{T}_{M}^{i}
\end{gathered}
$$




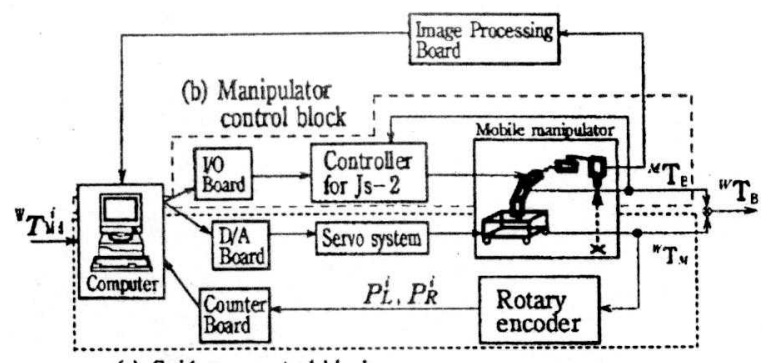

(a) Guidance control block

Fig. 3 Block Diagram of Our Hardware

The error $\Delta \hat{T}_{M}^{i}$ of the estimation is described by Eq.(14) using Eqs.(11) through (13).

$$
\Delta \hat{T}_{M}^{i}=\Delta T_{M}^{i}\left(\Delta T_{G}^{i}\right)^{-1}
$$

Here, the actual position/orientation and the estimated one are different. Then, it is necessary to correct the difference when the mobile manipulator reaches at a destination, i.e., before a next guidance. Therefore, the position/orientation is corrected by:

$$
\Delta \hat{T}_{M}^{0}=\Delta T_{M}^{0}\left(\Delta T_{G}^{0}\right)^{-1}
$$

where the symbol "0" denotes time of the beginning of the guidance. $\Delta T_{M}^{0}$ is obtained by a method shown in the following subsection and $\Delta T_{G}^{0}$ is given in the guidance controller. Then, $\Delta \hat{T}_{M}^{0}$ is determined.

\subsection{Detection of Position/Orientation}

A method of detecting the position/orientation of the mobile manipulator, in which a landmark is set on the flat plane and a hand-eye camera is faced to the plane vertically, is proposed in References [4],[5]. An error $\Delta T_{M}$ of the position/orientation, which is described by a homogeneous matrix by using the method in the references, is given by:

$$
\Delta T_{M}={ }^{M 0} T_{60}{ }^{60} T_{C 0} \Delta T_{C}{ }^{60} T_{C 0}^{-1}{ }^{M 0} T_{60}^{-1}
$$

where ${ }^{M 0} T_{60}$ is a posture of a mounted manipulator, ${ }^{60} \boldsymbol{T}_{C 0}$ is a relationship of the position/orientation between a hand and a camera and $\Delta T_{C}$ is an error of the position/orientation of the camera. From Eq.(16), $\Delta T_{M}$ is obtained by detecting $\Delta T_{C}$.

\section{Hardware}

Fig. 2 shows a photo of our constructed mobile manipulator and Fig. 3 shows a block diagram of the hardware. The mounted manipulator is a robot (Js-2) made by Kawasaki heavy industry and the mobile robot is made by acrylic resin boards. The mobile robot is traveled and steered by two different driving wheels, which are driven by two DC motors through metallic chains. In a guidance control block (a) of Fig.3, $P_{L}^{i}$ and $P_{R}^{i}$, which are pulse counts from the both driving wheel at time period $\Delta \tau$, are detected by rotary

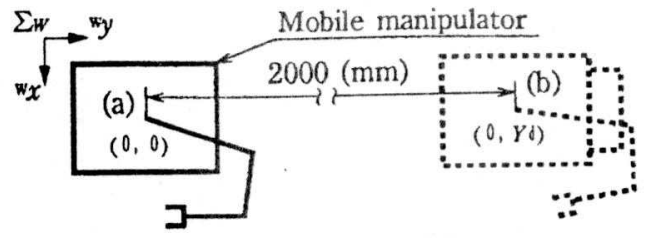

Fig. 4 Aerial view of an experiment

encoders and are inputted to a computer for the guidancc controller through a counter board. The computer calculates an estimated value ${ }^{W} \hat{T}_{M}^{i}$ from $P_{L}^{i}$ and $P_{R}^{i}$ and determines control values $V_{c}^{i+1}$ and $\omega_{c}^{i+1}$ to the each wheel. The desired angular velocities $V_{c}^{i+1}$ and $\omega_{c}^{i+1}$ are inputted to servo systems as desired volts through a D/A board. Then, the DC motors run and the mobile manipulator travels to a destination. In the manipulator control block (b), the desired posture of the mounted manipulator, which is for detecting an error of the position/orientation of the mobile manipulator at a destination, is inputted to the controller for the mounted manipulator (Js2) through a I/O board. Then, the manipulator moves to realize the posture.

\section{Gain Tuning}

From preliminary traveling experiments, it is obtained that $\Delta \hat{T}_{M}^{i}$ is a dominant parameter for the occurrence of $\Delta T_{M}^{i}$ and $\Delta T_{G}^{i}$ is small while navigating. $\Delta \hat{T}_{M}^{i}$ increases as the length of a traveling distance increases, because $\Delta \hat{T}_{M}^{i}$ is an integrated error of Eqs.(3) through (5). Thus, $\Delta \hat{T}_{M}^{0}$, which is obtained at a point for detecting a landmark, becomes maximum at a detecting point of a landmark. Then, an output value of initial guidance control is maximum just after a correction by Eq.(15). In this section, gains $k_{x}, k_{y}$ and $k_{\theta}$, which are gains of the guidance controller in which forces exerting to carrying objects do not exceed friction forces exerting them, are considered.

\subsection{Force/Torque Exerting Carrying Objects}

By focusing on that carrying objects do not move when a translational force $f_{S}$ and a torque $\tau_{S}$ exerting the object are smaller than friction forces exerting it, the gains of the guidance controller are decided. A translational force $f_{S}$ and a torque $\tau_{S}$ exerting the carrying object are given by:

$$
\begin{aligned}
& f_{S}=\left.m_{S}\right|^{\boldsymbol{W}} \ddot{\boldsymbol{P}}_{S} \mid \\
& \tau_{S}=\left|\boldsymbol{I}_{S}{ }^{W} \dot{\boldsymbol{\omega}}_{S}+{ }^{W} \boldsymbol{\omega}_{S} \times\left(\boldsymbol{I}_{S}{ }^{W} \boldsymbol{\omega}_{S}\right)\right|
\end{aligned}
$$

where $m_{S}$ is a mass of the carrying object, ${ }^{W} \ddot{\boldsymbol{P}}_{S}$ and $\boldsymbol{I}_{S}$ are a translational acceleration and an inertia tensor of the carrying object, respectively, ${ }^{W} \omega_{S}$ and ${ }^{W} \dot{\omega}_{S}$ are an angular velocity and acceleration exerting the carrying object, the symbol " $x$ " is a cross product of vector and the symbol " $|\cdot| "$ is Euclid norm. In the present paper, 
only a rotational motion around $W_{z}$ axis is considered on the assumption of a flat plane, then ${ }^{W} \boldsymbol{\omega}_{S}=\left[0,0, \omega_{S, z}\right]^{T}$. Thus, ${ }^{W} \boldsymbol{\omega}_{S} \times\left(I_{S}{ }^{W} \boldsymbol{\omega}_{S}\right)=\mathbf{0}$ and Eq. (18) becomes Eq.(19).

$$
\tau_{S}=\left|I_{S}{ }^{W} \dot{\omega}_{S}\right|
$$

When the carrying object is stationary while traveling, the translational acceleration ${ }^{W} \ddot{\boldsymbol{P}}_{S}$ and the angular acceleration ${ }^{W} \dot{\omega}_{S}$ of the object are calculated by:

$$
\begin{aligned}
{ }^{W} \ddot{\boldsymbol{P}}_{S} & ={ }^{W} \ddot{\boldsymbol{P}}_{M}+{ }^{W} \dot{\boldsymbol{\omega}}_{M} \times{ }^{W} \boldsymbol{R}_{M}{ }^{M} \boldsymbol{P}_{S} \\
& +{ }^{W}{ }^{\omega_{M}} \times\left[{ }^{W} \omega_{M} \times\left({ }^{W} \boldsymbol{R}_{M}{ }^{M} \boldsymbol{P}_{S}\right)\right](20) \\
{ }^{W} \dot{\omega}_{S} & ={ }^{W} \dot{\boldsymbol{\omega}}_{M}
\end{aligned}
$$

where ${ }^{W} \ddot{\boldsymbol{P}}_{M}$ is a traveling acceleration, ${ }^{\boldsymbol{M}} \boldsymbol{P}_{S}$ is a position of the object on the mobile manipulator referring to $\mathbf{\Sigma}_{M},{ }^{W} \boldsymbol{R}_{M}$ is a transformation matrix of orientation between $\boldsymbol{\Sigma}_{W}$ and $\boldsymbol{\Sigma}_{M}$. In addition, when $n$ carrying objects exist on the mobile manipulator, a maximum exerting force influences to the object which locates at most far from the origin of $\boldsymbol{\Sigma}_{M}$. Therefore, ${ }^{M} \boldsymbol{P}_{S}$ is defined by:

$$
{ }^{M} \boldsymbol{P}_{S} \equiv\left\{{ }^{M} \boldsymbol{P}_{S i}\left|\max _{i=1, \cdots, n}\right|{ }^{M} \boldsymbol{P}_{S i} \mid\right\}
$$

Then, $f_{S}$ and $\tau_{S}$ are obtained by substituting ${ }^{W} \ddot{\boldsymbol{P}}_{S}$, which is determined by Eqs.(20) through (22), into Eqs.(17) and (19).

\subsection{Friction Force Exerting Carrying Objects}

Next, ${ }^{W} f_{S, \text { max }}$ and ${ }^{W} \tau_{S, \text { max }}$ exerting a carrying object just before the beginning of that the carrying object starts translational and rotational motions are considered and are given by:

$$
\begin{aligned}
{ }^{W} f_{S, \text { max }} & =-\left({ }^{W} \boldsymbol{f}_{S} /\left.\right|^{W} f_{S} \mid\right) \mu m_{s} g \\
{ }^{W} \tau_{S, \text { max }} & =\left[0,0, \int_{S} \Delta f_{f} r d s\right]^{T}
\end{aligned}
$$

where $\mu$ is a static friction coefficient, $g$ is a gravitational acceleration, $r$ is a distance between a center of the carrying object and a minute area $d s, \Delta f_{f}$ is a friction force at $d s$. When the force/torque exerting the carrying object exceeds the above mentioned ${ }^{W} f_{S, \max }$ and ${ }^{W} \tau_{S, \max }$, the object occurs translational and rotational slip motions. Thus, the force/torque in Eqs.(23) and (24) is expressed with the symbol "max" and the force/torque is a maximum admissible exerting force/torque. Thercfore, when $f_{S}$ and $\tau_{S}$ given by Eqs.(17) and (19) satisfy Eq.(25), the carrying object do not slip.

$$
f_{S} \leq\left|{ }^{W} f_{S, \max }\right|, \quad \tau_{S} \leq\left.\right|^{W} \tau_{S, \max } \mid
$$

\subsection{Determination of Gains}

A range of error of the guidance is examined in order to determine predictive initial errors which are important parameters of deciding gains of the guidance controller. Fig. 4 shows an aerial view of an experiment. A desired trajectory is given straightly $(0,0)$ to $(0$,
$\left.Y_{d}\right)$ along ${ }^{W} y$ axis. In addition, a desired traveling speed $V_{d}^{i}$ is determined as a trapezoid pattern, which is; (1) $V_{d}^{i}=\alpha i \Delta \tau$ when a speedup section $\left(0 \leq \hat{y}^{i}<L_{a c c}, \quad L_{a c c}=V_{\text {const }}^{2} / 2 \alpha\right),(2) V_{d}^{i}=V_{\text {const }}$ when a constant section $\left(L_{a c c} \leq \hat{y}^{i}, L_{a c c}<L_{R}^{i}\right.$, $\left.L_{R}^{i}=Y_{d}-\hat{y}^{i}\right),(3) V_{d}^{i}=V_{\text {const }} \sqrt{L_{R}^{i} / L_{a c c}}=\sqrt{2 \alpha L_{R}^{i}}$ when a slowdown section $\left(L_{R}^{i}<L_{a c c}\right)$, where $V_{\text {const }}$ is a constant traveling speed, $L_{a c c}$ is a traveling distance for specdup and slowdown, $L_{R}^{i}$ is a remained distance referring to a desired position $Y_{d}$. In addition, $\alpha$ is an acceleration of the mobile manipulator and determined by $\alpha=V_{\text {const }} /\left\{T_{d}-\right.$ $\left.\left(Y_{d} / V_{\text {const }}\right)\right\}$.

The mobile manipulator traveled (a) to (b) with the desired traveling speed in condition that an initial error was not inputted, i.e., $X_{e}^{i}=Y_{e}^{i}=\theta_{e}^{i}=0$. Then, mean values of traveling errors, which are obtained by repeated travels, were $X_{e 0}=-0.012$ $\mathrm{m}, Y_{e 0}=-0.016 \mathrm{~m}$ and $\theta_{e 0}=0.856 \mathrm{deg}$. From the result, gains $k_{y}=0.07, k_{x}=0.006, k_{\theta}=0.25$, which do not produce slip motions of a carrying object, were obtained.

\section{Guidance Method with Limits}

\subsection{Traveling Condition for Preventing Slip}

A method, in which the mobile manipulator is navigated by decreasing traveling translational and angular accelerations when exerting forces to a carrying object exceed static friction forces exerting it, is considered. Equations (17), (19) and (25) lead:

$$
\begin{aligned}
& \left|{ }^{W} \ddot{P}_{S}\right| \leq\left|f_{S, \text { max }}\right| / m_{s} \\
& \left|{ }^{W} \dot{\omega}_{S}\right|=\left|\dot{\omega}_{S, z}\right| \leq r_{S, \text { max }} / I_{S, z}
\end{aligned}
$$

where $I_{S, z}$ is an inertia moment of the carrying object around ${ }^{W} z$ axis. Substituting $\left|{ }^{W} \ddot{\boldsymbol{P}}_{S}\right|$ of Eq.(20) into Eq.(26), conditions that the carrying object do not slip while traveling are described by Eqs.(27) and (28).

$$
\sqrt{\dot{V}^{2}+2\left(\dot{\omega} x_{S}-\omega^{2} y_{S}\right) \dot{V}+c} \leq\left|f_{S, \max }\right| / m_{s}
$$

where ${ }^{M} P_{S}=\left[x_{S}, y_{S}, z_{S}\right]^{T}, c=V^{2} \dot{\theta}^{2}+\left(\dot{\omega}^{2}+\right.$ $\left.\omega^{4}\right)\left(x_{S}^{2}+y_{S}^{2}\right)+2 V \dot{\theta}\left(\omega^{2} x_{S}+\dot{\omega} y_{S}\right)$.

\subsection{Recalculation of Control Output Values}

Here, in order to evaluate output values of a guidance control, a control translational speed $V_{c}^{i+1}$ and a control angular velocity $\omega_{c}^{i+1}$ obtained by Eqs. (9) and (10) are called infinite translational speed and angular velocity of a guidance control and are rewritten as $\tilde{V}_{c}^{i+1}$ and $\tilde{\omega}_{c}^{i+1}$, respectively.

When $\tilde{V}_{c}^{i+1}$ and $\tilde{\omega}_{c}^{i+1}$ are outputted by supposing no limits of translational and angular accelerations, approximate command values of the translational acceleration $\dot{\tilde{V}}_{c}^{i+1}$ and the angular acceleration $\dot{\tilde{\omega}}_{c}^{i+1}$ at next $\Delta \tau$ are written as:

$$
\frac{\tilde{V}_{c}^{i+1}-\bar{V}_{c}^{i}}{\Delta \tau} \equiv \dot{\tilde{V}}_{c}^{i+1}, \frac{\tilde{\omega}_{c}^{i+1}-\vec{\omega}_{c}^{i}}{\Delta \tau} \equiv \dot{\tilde{\omega}}_{c}^{i+1}
$$

where $\bar{V}_{c}^{i}$ and $\bar{\omega}_{c}^{i}$ are one past sampling values. 


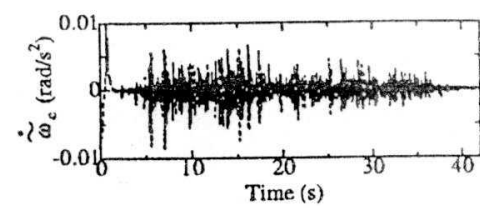

(a) $\dot{\tilde{\omega}}_{c}$ (free)

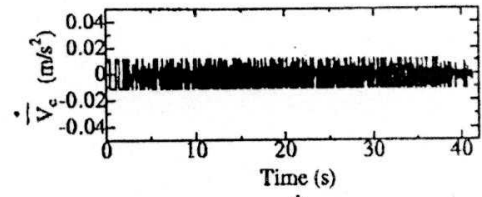

(d) $\dot{\bar{V}}_{c}$ (limited)

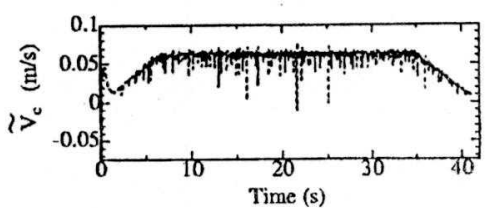

(g) $\tilde{V}_{c}$ (free)

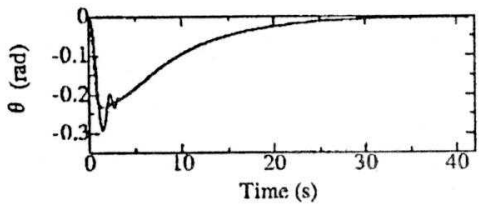

(j) $O(0 \sim 40[\mathrm{~s}])$

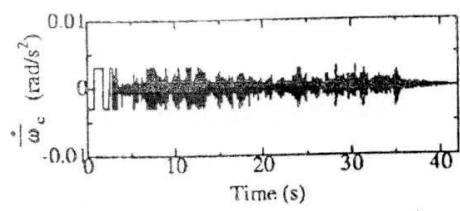

(b) $\dot{\bar{\omega}}_{c}$ (limited)

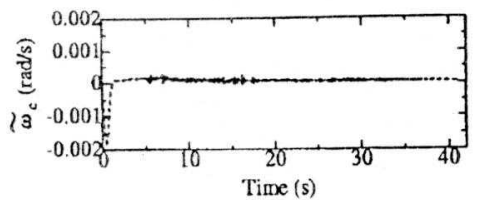

(e) $\tilde{\omega}_{c}$ (free)

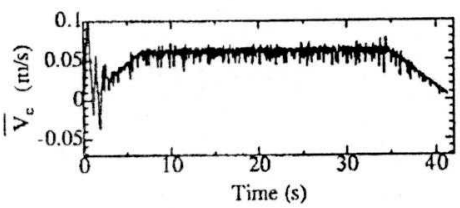

(h) $\bar{V}_{c}$ (limited)

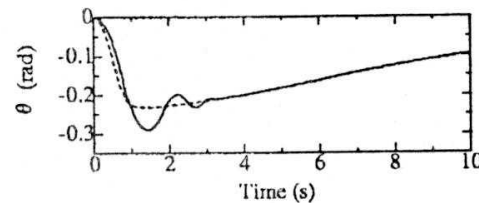

(k) $\theta(0 \sim 10[\mathrm{~s}])$

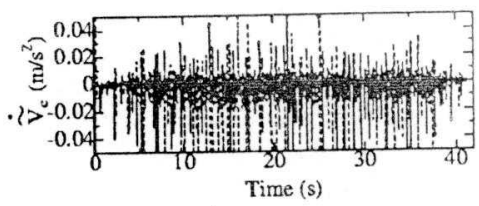

(c) $\dot{\tilde{V}}_{c}$ (free)

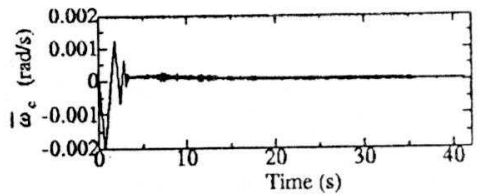

(f) $\bar{\omega}_{c}$ (limited)

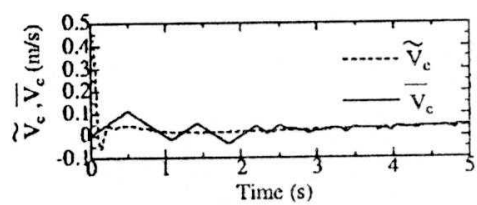

(i) $\tilde{V}_{c}, \bar{V}_{c}(0 \sim 5[\mathrm{~s}])$

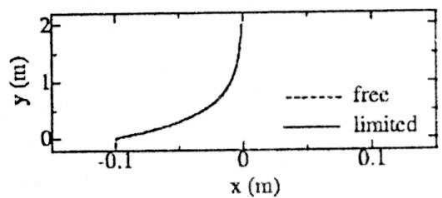

(l) Trajectory

Fig. 5 Results of Experiment

By using those $\dot{\tilde{V}}_{c}^{i+1}$ and $\dot{\tilde{\omega}}_{c}^{i+1}$, new commands of the translational acceleration $\dot{\bar{V}}_{c}^{i+1}$ and the angular acceleration $\dot{\bar{\omega}}_{c}^{i+1}$ are calculated in the following subsections.

\subsubsection{Limit of Angular Accelerations}

The $\dot{\tilde{\omega}}_{c}^{i+1}$ determined by Eq.(29) when a carrying object does not occur slip is:

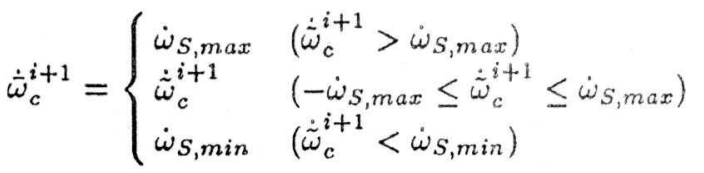

where $\dot{\omega}_{S, \text { max }}$ and $\dot{\omega}_{S, \text { min }}$ are an upper and a lower limits of an angular acceleration exerting the carrying object. $\dot{\omega}_{S, \max }$ and $\dot{\omega}_{S, \text { min }}$ are a boundary of $\dot{\omega}_{S, z}$ obtained by Eq.(27) and $\dot{\omega}_{S, \text { min }}=-\dot{\omega}_{S, \text { max }}$. A new guidance control output angular vclocity $\bar{\omega}_{c}^{i+1}$ is determined by Eq.(31) using the $\dot{\bar{\omega}}_{c}^{i+1}$. The $\bar{\omega}_{c}^{i+1}$ is a command of angular velocity with a limit of angular acceleration.

$$
\bar{\omega}_{c}^{i+1}=\dot{\bar{\omega}}_{c}^{i+1} \Delta \tau+\omega_{c}^{i}
$$

\subsubsection{Limit of Translational Accelerations}

When a translational acceleration $\dot{V}$ of a mobile manipulator exists between two solutions $\dot{V}_{\text {min }}$ and $\dot{V}_{\max }$, a carrying object does not slip, and otherwise, when $\dot{V}$ exceeds $\dot{V}_{\max }$ or $\dot{V}_{\min }$, the object slips. Here, an upper limit $\dot{V}_{\max }$ and a lower limit $\dot{V}_{\min }$ are obtained by solving Eq. (28) for $\dot{V}$ and are:

$$
\begin{aligned}
\dot{V}_{\text {max }}= & -x_{S} \dot{\bar{\omega}}_{c}^{i+1}+y_{S}\left(\bar{\omega}_{c}^{i+1}\right)^{2}+\sqrt{b} \\
\dot{V}_{\text {min }}= & -x_{S} \dot{\bar{\omega}}_{c}^{i+1}+y_{S}\left(\overline{\bar{\omega}}_{c}^{i+1}\right)^{2}-\sqrt{b} \\
b= & \left(f_{S, \text { max }}^{2} / m_{S}^{2}\right)-2 x_{S} y_{S} \dot{\bar{\omega}}_{c}^{i+1}\left(\bar{\omega}_{c}^{i+1}\right)^{2} \\
& -\left(V^{i} \dot{\theta}^{i}\right)^{2}-y_{S}^{2}\left(\dot{\vec{\omega}}_{c}^{i+1}\right)^{2}-x_{S}^{2}\left(\bar{\omega}_{c}^{i+1}\right)^{4} \\
& -2 V^{i} \dot{0}^{i}\left\{x_{S}\left(\bar{\omega}_{c}^{i+1}\right)^{2}+y_{S} \dot{\bar{\omega}}_{c}^{i+1}\right\}
\end{aligned}
$$

where an angular velocity $\omega$ and an angular acceleration $\dot{\omega}$ are $\bar{\omega}_{c}$ and $\dot{\bar{\omega}}_{c}$ obtained by the previous subsection, respectively.

A command of translational acceleration which does not slip the carrying object using the above equations is given by:

$$
\dot{\bar{V}}_{c}^{i+1}= \begin{cases}\dot{V}_{\max } & \left(\dot{\tilde{V}}_{c}^{i+1}>\dot{V}_{\max }\right) \\ \dot{\tilde{V}}_{c}^{i+1} & \left(\dot{V}_{\min } \leq \dot{\tilde{V}}_{c}^{i+1} \leq \dot{V}_{\max }\right) \\ \dot{V}_{\min } & \left(\dot{\tilde{V}}_{c}^{i+1}<\dot{V}_{\min }\right)\end{cases}
$$

A new guidance control output velocity $\bar{V}_{c}^{i+1}$ is calculated by Eq.(36) using the $\dot{\bar{V}}_{c}^{i+1}$. The $\bar{V}_{c}^{i+1}$ is a command of traveling speed with a limit of a translational acceleration.

$$
\bar{V}_{c}^{i+1}=\dot{\bar{V}}_{c}^{i+1} \Delta \tau+V_{c}^{i}
$$




\section{Experiments}

The validity of the proposed guidance method is evaluated through real experiments. The desired traveling course is same as the course shown in Fig. 4 used in Subsection 4.3. The gains were set as $k_{x}=0.007, k_{y}=0.0006, k_{\theta}=0.025$. 'The initial errors were set as $X_{e}^{0}=-0.10 \mathrm{~m}, Y_{e}^{0}=-0.02$ $\mathrm{m}$ and $\theta_{e}^{0}=0.0 \mathrm{deg}$, which are larger than the obtained errors in Subsection 4.3, i.e., the unexpected larger errors. The trapezoid pattern of desired traveling speed was set as $V_{\text {const }}=0.06$ $\mathrm{m} / \mathrm{s}, T_{d}=40 \mathrm{~s}$ and $\alpha=9.0 \times 10^{-3} \mathrm{~m} / \mathrm{s}$. In addition, $\left|{ }^{W} \boldsymbol{f}_{S, \text { max }}\right|=f_{S, \text { max }}=1.12 \mathrm{~N}$ and $\left|{ }^{W} \tau_{S, \text { max }}\right|=\tau_{S, \text { max }}=0.6 \times 10^{-3} \mathrm{Nm}$ were obtained through preliminary experiments.

Figs. $5(\mathrm{a})$ and (c) show $\dot{\tilde{\omega}}_{c}$ and $\dot{\tilde{V}}_{c}$ which are translational and rotational accelerations of the mobile rnanipulator without limits of accelerations. The figures show that those accelerations became large at the beginning of the guidance control. In contrast, it is shown in (b) and (d) that the limited accelerations $\dot{\bar{\omega}}_{c}$ and $\dot{\bar{V}}_{c}$ did not exceed upper and lower maximum value by setting limits for each values. In (e), $\tilde{\omega}_{c}$ changed on a large scale at the beginning of guidance around $1 \mathrm{~s}$ and then converged to zero, in which accelerations are not limited. On the other hand, $\tilde{\omega}_{c}$ oscillated till $5 \mathrm{~s}$ and then converged to zero in (f) with a limit. Fig. $5(\mathrm{~g})$ shows the traveling speed $\tilde{V}_{c}$ with no limit and shows that a traveling speed changed on a large scale at the beginning of the guidance control till $1 \mathrm{~s}$ and corresponded to the desired speed with vibrations. However, in (h) with a limit, $\bar{V}_{c}$ oscillated till $3 \mathrm{~s}$ and then corresponded to the desired speed. The close-up of $\bar{V}_{c}$ and $\tilde{V}_{c}$ at 0 to 5 $\mathrm{s}$ is shown in (i). The figure indicates that the $\bar{V}_{c}$ oscillated comparing the $\tilde{V}_{c}$. Fig. $5(\mathrm{j})$ shows the response of a head angle of the mobile manipulator and $(\mathrm{k})$ shows the close-up at 0 to $10 \mathrm{~s}$. From (j) and $(\mathrm{k})$, it is shown that a head angle with limits changed slower than that with no limits. Fig. $5(l)$ shows that the traveled trajectory with limits and that with no limits were approximately same.

From the above results, the validity of the proposed method was shown. In addition, the mobile manipulator traveled with no slip of a carrying object using the proposed guidance method even when unexpected initial errors are given.

\section{Conclusions}

In the present paper,

(1) We proposed the guidance control method in which control translational and angular accelerations to the mobile manipulator are limited in order to make exerting forces to a carrying object from traveling translational and angular accelerations be less than friction forces exerting the object.

(2) It is shown that the mobile manipulator traveled with no slip of a carrying object through real experiments by using the proposed control method.

\section{REFERENCES}

[1] T.Fukuda, etc.. "Manipulator for Man-Robot Cooperation Work(3rd Report, Control Algorithm of Center of Gravity for Manipulator/Vehicles System)" Trans. of the Japan Society of Mechanical Engineers, 58-551 C, p.21522158, (1992), (in Japanese)

[2] M.Kurisu and T.Yoshikawa. "Trajectory Planning and Dynamic Control of a Mobile Manipulator" Trans. of the Japan Society of Mechanical Engineers, 62-596 C, p.1488, (1996), (in Japanese).

[3] Q.Huang, S.Sugano and I.Kato. "Stability Control for a Vehicle-Mounted Manipulator . Stability Evaluation Criteria and Manipulator Compensatory Motion-" J. of Society of Instrument and Control Engineers, Vol.31, No.7, p.861-870, (1995), (in Japanese)

[4] M.Minami, N.Fujiwara and H.Tsuge. "Position . Velocity Control of Autonomous Mobile Manipulator with Camera Feedback" J. of Robotics Society of Japan, Vol.11, No.2, p.263-271, (1993), (in Japanese).

[5] M.Hatano, M.Minami and T.asakura. "Control for Piling Up Blocks by Mobile Manipulators Experiments of Piling Up Blocks with Real System and Piling Up Accuracy-" The 15th Annual Conf. of Robotics Society of Japan, p.277-278, (1997), (in Japanese).

[6] Y.Kanayama, Y.Kimura, F.Miyazaki and T.Noguchi. "A Stable Tracking Control Method for an Autonomous Mobile Robot" Proc. of Int. Conf. on Rob. and Autom.,pp.384-389(1990)

[7] Zhang Y.L., Velindky S.A. and Feng X.. "On the Tracking Control of Differentially Steered Whecled Mobile Robots" J. of Dynamic Systems, Measurement and Control,Vol.119,pp.455461(1997)

[8] Fierro R. and Lewis F.L.. "Control of a Nonholonimic Mobile robot: Backstepping Kinematics into Dynamics" J. of Robotic Systems,Vol.14,No.3,pp.149-163(1997)

[9] T.Tsumura, N.Fujiwara and M.Hashimoto. "An Experimental System for Self-contained Posjtion and heading Measurement of Ground Vehicle" Int.Conf.on Advanced Robotics,pp. 269276(1983)

[10] J.Y.S.Luh, M.Walker and R.P.Paul. "On-Line Computational Scheme for Mechanical Manipulators" Trans.ASME,Journal of DAMC, 102, pp.69-76(1980)

[11] M.Minami, M.Sudare, S.Ueda and Y.Nakano. "Autonomous Guidance Method to Arbitrarily Curved Course with Homogeneous Matrix" J. of Robotics Society of Japan, Vol.8, No.4, p.421430, (1990), (in Japanese).

[12] Y.Nakamura. "Nonholonomic Robot Systems(Part 2:Motion Planning under Kinematic Nonholonomic Constaints)" Journal of Robotics Society of Japan, Vol.11, No.5, p.655-662, (1993), (in Japanese). 